\title{
Erratum to: Aggressive surgical resection after neoadjuvant chemoradiation therapy for locally advanced intrahepatic cholangiocarcinoma
}

\author{
Hiroyuki Kato - Masami Tabata - Motoyuki Kobayashi - Ichiro Ohsawa • \\ Masashi Kishiwada - Shugo Mizuno • Masanobu Usui · Hiroyuki Sakurai · \\ Shuji Isaji
}

Published online: 21 November 2009

(c) Springer 2009

Erratum to: Clin J Gastroenterol (2009) 2:351-354

DOI 10.1007/s12328-009-0102-z

Please note that there are errors in this article:

On pages 352 (right column) and 353 (left column), in the "Case report" section, figure citations should be renumbered as follows:

Now shows:

Should read:

Fig. 1

Fig. 2

Fig. 3
Fig. 1a

Fig. 1b

Fig. 2a
In addition, on page 353 (right column), also in the "Case report" section, lines 4-10 should read as follows, with the figure citations as shown here:

"The resected specimen revealed that the main tumor was a well-differentiated adenocarcinoma arising from the caudate lobe (Fig. 2b), whereas almost all neoplastic cells were replaced by extensive fibrosis, hyalinization, and hemosiderosis (Fig. 3a). Furthermore, dissected regional lymph nodes also showed marked fibrosis and no evidence of a viable tumor (Fig. 3b)."

The online version of the original article can be found under doi:10.1007/s12328-009-0102-z.

H. Kato $(\square) \cdot$ M. Tabata - M. Kobayashi - I. Ohsawa ·

M. Kishiwada - S. Mizuno - M. Usui · H. Sakurai - S. Isaji

Department of Hepatobiliary Pancreatic and Transplant Surgery,

Mie University Graduate School of Medicine,

Tsu, Mie 514-8507, Japan

e-mail: kato0719@clin.medic.mie-u.ac.jp 